\title{
Parkour y skateboard, intersubjetividad en las calles de la ciudad de Bogotá*
}

\author{
Parkour and skateboard, intersubjectivity in the streets \\ of the city of Bogotá
}

\author{
John Carlos Cortés-Murillo**
}

\section{Resumen}

Objetivo. Interpretar los sentidos y significados que subyacen en las relaciones que los practicantes de parkour y skateboard tejen dentro de su práctica y con los demás habitantes de la ciudad. Metodología. Este es un estudio fenomenológico que recurre a entrevistas de discusión y entrevistas semiestructuradas realizadas a grupos e individuos que hacen parte de las prácticas. Resultados. Los resultados indican que las relaciones intersubjetivas en la práctica se caracterizan por su horizontalidad, en ellas los saberes y experiencias valen por su carácter formativo y amistoso y no por la jerarquía. Dentro de las practicas la interrelación de los sujetos está circunscrita a la solidaridad, la responsabilidad, la motivación y cuidado del otro. Conclusión. Los procesos de subjetivación e intersubjetividad se alimentan del sí mismo de los otros, traceurs y skaters proyectan no solo sus propias subjetividades, sino que reflejan algunas formas de aquellos con los que confrontan.

Palabras clave: parkour, skate, intersubjetividad, prácticas urbanas.

\begin{abstract}
Objective: to interpret the senses and meanings that underlie the relationships that parkour and skateboard practitioners weave within their practice and with the other inhabitants of the city. Methodology: this is a phenomenological study that uses discussion interviews and semi-structured interviews conducted with groups and individuals that are part of the practices. Results: The results indicate that inter-subjective relationships in practice are characterized by their horizontality, in which knowledge and experiences are worth because of their formative and friendly nature and not by hierarchy. The interrelation of the subjects is limited to the solidarity, responsibility, motivation and care of the other within the practices. Conclusion: the processes of subjectivation and intersubjectivity feed on the self of others, traceurs and skaters project not only their own subjectivities, but also reflect some forms of those with which they confront.
\end{abstract}

Key words: parkour, skate, intersubjectivity, urban practices.

\footnotetext{
* Segundo artículo derivado del proyecto de investigación: Parkour, Skateboard y ciudad. Una mirada fenomenológica a sus practicantes y su vínculo con el espacio público urbano en Bogotá. El diseño metodológico corresponde al mismo que aparece en otro artículo derivado de la misma investigación y fue publicado en 2018 en Convergencia Revista de Ciencias Sociales

** Corporación Universitaria Cenda. Bogotá, Colombia. E-mail: jhon.cortes@cenda.edu.co

(D) orcid.org/0000-0001-8160-7979 Google Scholar
} 


\section{Introducción}

En la ciudad las formas de significación y representación convocan a la integración, pero también a la confrontación; el carácter compacto, sólido y estrecho, pero simultáneamente abierto y fluido de la urbe configura múltiples y cada vez más numerosas prácticas y formas de relacionarnos en ella. Estas obedecen a los procesos de transformación y organización endógena propios de toda sociedad, así como a las prácticas que dicho orden establece.

Entre las prácticas que confrontan constantemente con el orden urbano se encuentran el parkour y skateboard. Estas se destacan por su vistosidad, acogida entre los jóvenes y por su espíritu retador frente al dinamismo social y en especial, frente a las convenciones tradicionales del uso de los espacios públicos. La apropiación nómada que sus practicantes hacen de los espacios, el no estar inscritos en el orden institucional convencional, sumado al desconocimiento de las características particulares de cada práctica y de los significados que dichos sujetos le atribuyen a su actuar, representa para algunos habitantes de la ciudad las razones por las cuales confrontan sus maneras de proceder y olvidan comprender su innegable filosofía de alteridad.

Sin lugar a duda, estas prácticas y sus agentes reclaman un lugar preponderante en la vida de la ciudad, un espacio en el que las tensiones territoriales y simbólicas entre prácticas genere convenciones y tensiones frente a los comportamientos y relaciones entre sujetos que deben dar lugar a discutir el carácter valorativo y específico de las mismas, pues estas configuran las subjetividades que, articuladas entre sí, gestan el valor y sentido de vivir con otros en la ciudad. En este sentido, cada sujeto, cada habitante, resulta fundamental en la generación de formas particulares de apropiar y construir la socialidad urbana, por tanto, prácticas como el parkour y el skateboard hacen parte de esa red de relaciones que posteriormente se configura en un referente de quienes la viven y de aquellos que la contemplan desde afuera.

Frente a lo anterior, es necesario puntualizar que el surgimiento, consolidación y desarrollo de prácticas corporales alternativas como el parkour y skateboard en Bogotá no cuenta con suficiente ni precisa información, su aparición como hecho social, práctica recreativa o de ocio no se puede establecer con claridad. En Colombia, los antecedentes muestran cuatro estudios realizados en la capital referentes al parkour, Rotawisky (2013, 2015) y Cortés y Certuche (2013, 2015); los primeros, hechos desde un enfoque etnográfico, mientras los dos siguientes difieren en su perspectiva, uno, el más reciente corresponde a un análisis cualitativo sobre la relación del traceur - practicante de parkour - con los espacios de la ciudad y su valor pedagógico para la educación física, el otro se enfoca en las características sociodemográficas de los practicantes. En cuanto al skateboard, se encuentran los estudios realizados por Zuluaga y Vélez (2013), Cortázar (2015) y Patiño (2010) en ellos se describe la importancia de la práctica como manifestación cultural, identidad juvenil, postura política y alternativa a las problemáticas sociales, sin embargo y a pesar de estos referentes, se puede inferir que en el contexto nacional estas prácticas se han considerado menos importantes para la academia y el conocimiento que existe sobre ellas es limitado. 
Parkour y skateboard, intersubjetividad en las calles de la ciudad de Bogotá

Caso contrario ocurre en el panorama internacional, el número de estudios es significativo, autores como Redondo (2011), Gutiérrez y Rosales (2011), Scarnatto y Díaz (2010), Villagrán y Scarnatto (2013), Ferro (2011), Silva (2012), Carvalho y Pereira (2008), Pérez (2013), Montoro y Baena (2009), Caamaño (2007); Camino (2008, 2013), Vieira y De Sousa (2016), Márquez y Díez (2015), Blanco (2016), Machado (2014), Da Silva y Diniz (2014), Cornejo, Villalobos, Cerda y Cuadra (2006), Bermúdez (2008), Quinteros (2017) y Saraví (2007, 2012, 2014); realizaron diversos análisis desde enfoques cualitativos, cuantitativos, empíricos, simbólicos, sociológicos, pedagógicos, centrados en detallar las características técnicas, tecnológicas, organizativas, estructurales, políticas, y en ocasiones educativas, bien sea de los grupos o del accionar de sus practicantes; sin embargo, estos análisis no muestran una preocupación particular sobre la configuración de la subjetividad de los practicantes y mucho menos dan cuenta de la intersubjetividad endógena como factor determinante en las relaciones que se establecen con los demás. Bajo esta perspectiva, el derrotero de esta investigación considera que este tipo de prácticas representan para sus integrantes espacios de confrontación y dialogo en los que la intersubjetividad se gesta a partir de una filosofía cimentada en las experiencias que se dan en y con el espacio urbano, pero bajo el cuidado de sus pares.

\section{Del sí mismo a la intersubjetividad}

Desde su origen, el parkour y skateboard se han forjado en una estrecha simbiosis entre el cuerpo y el espacio, asumiendo este último como posibilitador de sus acciones. En este mismo sentido, las relaciones que se tejen entre practicantes también responden a dicha naturaleza, es decir, la destreza y fluidez corporal se convierte en el fundamento de las relaciones que los agrega y a la vez los diferencia de otros habitantes de la ciudad, es por esto que interpretar las experiencias gestadas en las relaciones que los traceurs y skaters — practicantes de parkour y skateboard - , establecen entre sí y con otros habitantes de la ciudad, implica antes que nada, aclarar el binarismo pensar-actuar que también hace parte de la cotidianidad de sus prácticas. Sin estas ideas, cualquier intento por conocer las intenciones, acciones y sentidos del sujeto queda reducido al determinismo objetivo que anula la persona como realidad configurante.

Frente al pensamiento, vale decir que este se expresa como actividad consciente, identificando los objetos que hacen parte del espacio-tiempo, es decir, objetos perceptibles y relacionales que están ahí, que se mueven, que aparecen y desaparecen, que se desgastan o que perduran (Cortes, Perea y Sarmiento, 2019). Esto no solo corresponde a la dimensión sensitiva del sujeto, sino también a lo que deriva de allí, es decir, a todas aquellas imágenes, ideas, representaciones, interrogantes y demás juicios formados sobre ellos y sus cambios. Así mismo, estas construcciones mentales son objeto de nuevas reflexiones que el sujeto hace para sí mismo, en este sentido, el sujeto es realidad sensible para sí, pero también es signo y significado, es representación, es código y lenguaje, en otras palabras, ante una situación cualquiera, el sujeto interactúa con el entorno y consigo mismo no solo como realidad corpórea sino como configuración simbólica. En consecuencia, el sujeto es autorreferente, se 
proyecta, construye y recrea en sus pensamientos y acciones, es consciente de sí, se percibe y comunica; por ello Schutz (1932) dirá que "el significado es intersubjetivo", viene de otros sujetos y de ellos se proyecta.

Por lo anterior, es importante aclarar que las vivencias en sí y no todas ellas son portadores de significado, este último es el resultado de un acto consciente e intencional sobre el mundo, de ahí que los motivos de las acciones humanas - motivo para y motivo porque- tal como los define Schutz (1932), sean la fuerza generativa y explicativa de las experiencias y acciones de todo sujeto consciente. El motivo para es el acto mismo proyectado en el tiempo, es decir, el sujeto realiza una acción que se sostiene o suspende en el tiempo por cuenta de sus intenciones o deseos, en otros términos, estos motivos expresan hasta dónde -temporal, espacial y emocionalmente--, se quiere llegar, dicho de otro modo, las acciones tienen finalidades. El motivo porque se refiere a hechos pasados cargados de sentido que han dejado huella y que proyectan los actos del sujeto como pauta de comportamiento consciente y reflexivo; de allí que solo este tipo de vivencias -las reflexivas- sean significativas y comunicantes en tanto portan los significados de lo vivido, en otros términos, existen razones y experiencias que originan las acciones. De acuerdo con Hernández y Galindo (2007), “el significado se encuentra en la relación de los actores con los objetos, y en esta relación el lenguaje resulta esencial, pues gracias a él, el mundo externo es ordenado" (p. 232). Es así como el significado se constituye intersubjetivamente, por ello, Schutz (1932) distingue dos tipos de significados: objetivos y subjetivos. El primero alude a contextos socio-culturales en los que el significado se comparte colectivamente, esto como resultado del segundo tipo de significado que refiere a los procesos de la conciencia individual que se relaciona con la realidad exterior, es decir con las cosas del mundo, con el mundo de las cosas -sus relaciones-, y con los significados del mundo, así, la experiencia del sí mismo y de su vínculo con los referentes exteriores, configuran el teatro de la intersubjetividad, una matriz de egos trascendentales para otros (Husserl como se citó en Hoyos, 1981).

\section{Intersubjetividad}

Las relaciones intersubjetivas corresponden a los vínculos entre la consciencia de sí con las consciencias de otros, es decir, como una manifestación de la existencia del ser y los significados que porta. En este sentido, el sujeto se manifiesta como signo, como grafía de una realidad que pertenece y proviene de la propia persona. Signos y acciones son comunicación, emergen de la intención, de la interacción con los otros, del lenguaje y sentido que allí se gesta, con el mundo en el que se proyecta, teje relaciones que muchas veces se naturalizan y se pierden en lo más profundo de la consciencia, "constituir al otro es mostrar cómo el sentido yo, que nace con la toma de conciencia de mi existencia como núcleo de sentido, se comunica a los otros y me permite decir que ellos son también egos” (López, 1994, p. 39). Así, el sí mismo es el punto de partida hacia la intersubjetividad que está mediada por la intención que brota de las experiencias individuales y colectivas, por lo tanto "la relación-nosotros se caracteriza por una 
Parkour y skateboard, intersubjetividad en las calles de la ciudad de Bogotá

relación hacia el tú que es la forma universal en el que el otro es experimentado en persona” (Schutz y Luckmann, 1973, p. 62).

Esta relación-nosotros no es otra cosa que la interacción de las intencionalidades, es decir, conductas que se proyectan, que pretenden ser o comunicar, en este sentido, en la acción se ha ideado previamente; tras de ella se encuentra la anticipación y tras de la anticipación las experiencias, por tanto, toda actividad humana conjuga dialécticamente sucesos del aquí y el ahora con las determinaciones sociales y subjetivas de los actores. Al proyectar las acciones, al cargarlas de intencionalidad, el sujeto se hace consciente de sí mismo y sus motivos, realidad que también percibe en los otros. Así, en el mundo social cada sujeto existe en la medida que se relaciona con las intencionalidades de los otros, es decir, mis semejantes, reflejos de lo que constituyen mis proyecciones. Mediante todas estas formas se instituye una multiplicidad de relaciones que componen la conciencia colectiva y cultural.

Frente a lo anterior, es importante considerar que el sujeto se agrega y hace parte y existe en una estructura de relaciones, las reproduce y resignifica de tal manera que interioriza el código social y revalora la clave de sentido que allí se gesta; por tanto, las relaciones intersubjetivas involucran una consciencia individual que remite a otras consciencias individuales exteriores. En este proceso, el lenguaje, la memoria y los relatos cotidianos son fundamentales, mediante ellos ocurre la conexión de las consciencias y aparecen los significados por equivalencia; en consecuencia, el mundo se revela como experiencia.

El lenguaje, la memoria y los relatos cotidianos son medios y fines, contienen y permiten la construcción de los significados; asimismo, estos elementos son dinamizadores de las interacciones entre sujetos que dan lugar a prácticas en las que, confrontar, dialogar o simpatizar entre sujetos implica una postura ética que reclama su presencia, lo contrario significa la invisibilización, la negación del otro trae consigo la reducción del sí mismo, de acuerdo con Lévinas (1987), el cara a cara es en esencia una relación y postura ética que refleja al sí mismo como libertad, posibilidad, diferencia y alteridad.

\section{Diseño metodológico}

Esta es una investigación fenomenológica, Moreira (2004) y Leal (1997), que interpreta la intersubjetividad de los practicantes de skate y parkour en Bogotá. La recolección de la información se efectuó mediante entrevistas grupales de discusión y entrevistas individuales semi-estructuradas, lo cual permite establecer puentes entre el mundo colectivo y subjetivo de los practicantes. Para la captura de la información y atendiendo a las particularidades de cada práctica se diseñaron y validaron cuatro guiones de entrevista: dos para entrevistas grupales y dos para entrevistas individuales, cuyo contenido y forma los validaron tres expertos en investigación cualitativa y una prueba piloto. 


\section{Los participantes}

Los participantes pertenecen a agrupaciones de parkour y skateboard que hacen uso de los espacios públicos - calles o skatepark- de la ciudad. Se trata de grupos que se reúnen periódicamente y presentan algún grado de organización y estructura formal o informal. Su vinculación se hizo mediante cuatro criterios que garantizan el desarrollo del trabajo: a) mostrar interés en participar de la investigación, b) que los participantes contaran con más de cinco años de práctica, c) realizar la práctica con frecuencia no menor a cuatro horas por semana, d) la relevancia de los casos de acuerdo con los planteamientos teóricos y las expectativas para el trabajo de campo. Estos aspectos garantizan el vínculo permanente del sujeto con la práctica $\mathrm{y}$, en consecuencia, la existencia de una relación que constituye el carácter subjetivo y los significados objeto de análisis, en otro sentido, es importante aclarar que en los grupos que aceptaron participar de la investigación, las mujeres no hacían parte de estos, aunque las alusiones a ellas fueron expuestas por los practicantes entrevistados.

\section{Trabajo de campo}

En el trabajo de campo se realizaron cuatro entrevistas grupales de discusión por cada práctica -4 grupos de parkour y 4 de skate-, dichas entrevistas se constituyen de tres encuentros para cada una. Los grupos los constituyeron cinco practicantes, que, a partir de su importancia y liderazgo, fueron elegidos por votación entre sus miembros. Lo anterior corresponde a 24 sesiones de trabajo con un promedio de 40 minutos de grabación para cada sesión, esto representa 960 minutos de registro.

Tabla 1. Grupos y fechas de realización de los encuentros de entrevista.

\begin{tabular}{lcccc}
\hline \multicolumn{5}{c}{ Parkour } \\
\hline Encuentro 1 & Frupo A & Grupo B & Grupo C & Grupo D \\
Encuentro 2 & Feb 23 de 2016 & Mar 30 de 2016 & May 25 de 2016 & Jul 27 de 2016 \\
Encuentro 3 & Mar 9 de 2016 & Abr 11 de 2016 & Jun 14 de 2016 & Ago 10 de 2016 \\
\hline & & Skateboard & & \\
\hline Encuentro 1 & Feb 16 de 2016 & Mar 24 de 2016 & Abr 19 de 2016 & Jun 7 de 2016 \\
Encuentro 2 & Mar 2 de 2016 & Mar 29 de 2016 & May 10 de 2016 & Jun 16 de 2016 \\
Encuentro 3 & Mar 15 de 2016 & Abr 8 de 2016 & May 18 de 2016 & Jul 13 de 2016 \\
\hline
\end{tabular}

Fuente: elaboración propia (2017). 
Parkour y skateboard, intersubjetividad en las calles de la ciudad de Bogotá

En cuanto a las entrevistas individuales se realizaron 24, del mismo modo cada entrevista se constituye de tres encuentros con cada sujeto, lo cual representa un total de 72 sesiones. En promedio cada encuentro tuvo una duración de 36 minutos de registro. El esquema de tres encuentros por cada entrevista se diseñó con el propósito de enfocar y ajustar paulatinamente la indagación a medida que se recababa la información. Para esto, las transcripciones de la primera y segunda sesión de trabajo se sometieron a lectura analítica o inferencial, lo cual permitía analizar e identificar aspectos de interés que posteriormente generaron nuevos interrogantes resueltos en los encuentros consecuentes. En cuanto a las edades y participantes de las entrevistas individuales, en cada grupo se escogieron los tres practicantes que contaban con el mayor número de años de experiencia. Para los grupos de parkour, practicantes de grupo A - P4, P2, P3, grupo B - P5, P3, P2, grupo C - P1, P5, P2, grupo D - P1, P5, P2; para los grupos de skateboard, grupo A - P1, P2, P5, grupo B - P2, P3, P5, grupo C - P2, P3, P4, grupo D - P1, P2, P3, tal como se muestra en la tabla 2. Dos semanas después de culminar las entrevistas grupales de discusión se inició el desarrollo de las entrevistas individuales entre agosto de 2016 y febrero de 2017. Todos los entrevistados -individuos y miembros de gruposson hombres. Las edades y años de experiencia (AE) de los participantes (P) de las entrevistas se distribuyen de la siguiente manera:

Tabla 2. Edades y años de experiencia de los practicantes.

\begin{tabular}{lcccccccccc}
\hline \multicolumn{10}{c}{ Parkour } \\
\hline & P 1 & AE & P 2 & AE & P 3 & AE & P 4 & AE & P 5 & AE \\
Grupo A & 18 & 6 & $\underline{\mathbf{2 1}}$ & 6 & $\underline{\mathbf{2 2}}$ & 6 & $\underline{\mathbf{2 9}}$ & 12 & 20 & 5 \\
Grupo B & 25 & 5 & $\underline{\mathbf{2 5}}$ & 5 & $\underline{\mathbf{2 3}}$ & 6 & 20 & 5 & $\underline{\mathbf{3 2}}$ & 7 \\
Grupo C & $\underline{\mathbf{2 3}}$ & 5 & $\underline{\mathbf{2 0}}$ & 6 & 19 & 5 & 19 & 5 & $\underline{\mathbf{1 8}}$ & 7 \\
Grupo D & $\underline{\mathbf{3 4}}$ & 14 & $\underline{\mathbf{3 0}}$ & 6 & 23 & 5 & 20 & 6 & $\underline{\mathbf{2 1}}$ & 9 \\
\hline & & & \multicolumn{10}{c}{ Skateboard } & & & & & \\
\hline Grupo A & $\underline{\mathbf{2 7}}$ & 7 & $\underline{\mathbf{2 2}}$ & 11 & 23 & 6 & 32 & 6 & $\underline{\mathbf{3 1}}$ & 9 \\
Grupo B & 20 & 5 & $\underline{\mathbf{3 9}}$ & 16 & $\underline{\mathbf{2 5}}$ & 9 & 34 & 6 & $\underline{\mathbf{2 9}}$ & 9 \\
Grupo C & 16 & 5 & $\underline{\mathbf{1 9}}$ & 5 & $\underline{\mathbf{2 4}}$ & 6 & $\underline{\mathbf{2 6}}$ & 6 & 17 & 5 \\
Grupo D & $\underline{\mathbf{3 0}}$ & 12 & $\underline{\mathbf{2 7}}$ & 9 & $\underline{\mathbf{2 5}}$ & 5 & 23 & 5 & 18 & 5 \\
\hline
\end{tabular}

Fuente: elaboración propia (2017).

Frente al número de participantes involucrados desde el inicio hasta el final del estudio, seis abandonaron -2 de parkour y 4 de skate-, debido a compromisos en competencias fuera del país. Los abandonos se dieron en la fase de entrevistas individuales, específicamente en la última sesión de trabajo. En cuanto a la captura de información, el registro fue hecho mediante grabadora de voz, previa autorización y firma de consentimiento informado y reserva de identidad de los entrevistados. 


\section{Esquema de análisis}

Para la organización, tratamiento y análisis de la información se determinaron tres momentos: 1) corresponde a la codificación axial de las entrevistas (Strauss y Corbin, 2002). El propósito de este paso es identificar en las transcripciones aquellas declaraciones asociadas a las relaciones intersubjetivas; 2) en este momento se diseña una matriz de doble entrada en la que se clasifica la información considerando la relación sujeto-otros como caso y las técnicas de recolección de información como variables, esta estructura permite identificar las diferencias entre las declaraciones individuales y colectivas, y permite decantar la carga subjetiva, influencia y rasgos discursivos de los practicantes, 3) la información clasificada se somete a codificación abierta (Coffey y Atkinson, 2003), con el propósito de elaborar un descripción interpretativa a partir de la identificación y relación de elementos recurrentes, de los énfasis y disonancias discursivas, de los tipos de experiencia, y de los significados que los practicantes atribuyen a sus relaciones y experiencias.

Para validar la información se realizaron dos procesos, el primero consistió en seleccionar, agrupar y enviar a cada entrevistado un documento con todas las respuestas que suministró. Este documento se estructuró a partir de tres elementos: 1) la pregunta que se hizo en la entrevista, 2) la respuesta del entrevistado, 3) una casilla de observaciones sobre la información provista en caso de querer aclarar la respuesta. En cuanto al segundo proceso, corresponde a la valoración de las descripciones teóricas por parte de los entrevistados. Para esto se hizo el envío de las descripciones interpretativas junto con un cuestionario de cinco preguntas en escala Likert, con el propósito de establecer el grado de conformidad entre los participantes de la investigación. Para identificar las declaraciones de los entrevistados dentro de los resultados, se ha definido en siguiente esquema y sus combinaciones: EI=entrevista individual, $\mathrm{EG}=$ entrevista grupal, $\mathrm{PP}=$ practicante parkour, $\mathrm{PS}=$ practicante skate, $\mathrm{G}=$ grupo.

\section{Resultados}

\section{Parkour: nosotros los traceurs y la armonía}

En el parkour los grupos de practicantes definen las relaciones intersubjetivas entre pares como relaciones de familia, estas se manifiestan en fuertes lazos de amistad construidos durante la práctica y usando redes sociales digitales, en el compartir conocimientos sobre las técnicas de movimiento que se realizan ante sus pares y que son objeto de crítica para depurarlas a medida que las emplazan. Cuando se práctica conjuntamente, los deseos individuales siempre están acompañados de motivaciones y sugerencias de sus pares, enfatizando en: inténtelo y sea persistente. 
Parkour y skateboard, intersubjetividad en las calles de la ciudad de Bogotá

Circula entre los practicantes un espíritu de gusto por lo que se hace, en este sentido, el amor por la práctica es la forma por excelencia para abrir la puerta a otros sujetos, de allí que se pretenda compartir amistosamente la esencia de moverse libre y estéticamente, "invité a un primo a practicar, él se metió a esto porque yo fui el que le insistí"(EI-PP1-GD, comunicación personal, 5 de agosto de 2016). El sentido de familiaridad no solo hace parte de la lógica organizativa de cada grupo, sino que también se manifiesta entre distintos grupos.

Cuando alguien ingresa a un grupo aprende lo básico por cuenta de sus miembros, con la adquisición de estas habilidades debe explorar los límites y las posibilidades de sí mismo, "vi como hacía las acrobacias y quise intentarlas, entonces fui a un parque cerca de mi casa y empecé a intentar dándome golpes" (EI-PP3-GB, comunicación personal, Setiembre 17 de 2016); para enseñar-aprender debe construirse una relación profunda de confianza, pues el parkour implica exponerse a riesgos físicos, "en el parkour, llevar a una persona a hacer un salto, a que se supere, a lograr una meta, nos une, entonces ocurre la conexión entre la persona que lo cuida y la persona que lo realiza”(EG-PP2-GB, comunicación personal, 30 de marzo de 2016); por tanto, el cuidado y compañía de sus compañeros hasta lograr cierto grado de autonomía es clave para sentirse en familia. Dicha autonomía se refleja en el arrojo consciente y calculado, en el cuidado oportuno y responsable con sus pares, y en vínculo profundo con la práctica, expresado en su constancia y permanencia, "no siempre todos pueden entrenar, entonces cuando mi grupo no entrena yo busco otros grupos” (EI-PP2-GB, comunicación personal, 3 de septiembre de 2016). Para aprender parkour no hay una metodología de enseñanza o aprendizaje definida, tan solo existen la demostración y los intentos, los ajustes y los consejos. Todo cambia a medida que se ejecutan los trucos, lo contrario significa una limitación de la práctica que desdibuja las posibilidades creativas y exploratorias en los movimientos.

Una de las motivaciones más fuertes de la práctica es la exaltación y reconocimiento de los progresos técnicos que posteriormente aportan al conocimiento de sus pares. La exhibición de los aprendizajes técnicos ante sus compañeros implica ganar confianza no solo ante los demás practicantes que continuamente alientan, “yo realicé los mejores trucos ese día, todo el mundo me aplaudía fue inolvidable ese día” (EG-PP1-GC, comunicación personal, 5 mayo de 2016); sino ante los retos que le impone el obstáculo y la creación o adecuación del gesto, de este modo cada sujeto aporta al grupo, configura un estilo y dinamiza sus relaciones al punto de generar lazos de confianza que le conduce a sentirse responsable de sus compañeros.

Cuando se aprende, las caídas y equivocaciones técnicas y en especial el impulso emocional se aprovecha coyunturalmente para enfrentar la situación, "yo creo que el cuerpo del traceur es como el artista que tiene un pincel y en un lienzo plasma su imaginación, probando, trazando, equivocándose" (EG-PP5-GD, comunicación personal, 7 de julio de 2016). En cuanto a los errores, rápidamente se transforman en oportunidades, que fortalecidas por el aliento recibido de los demás, enarbolan el espíritu persistente de la práctica. Quien quiera saber y aprender, 
solo debe acercarse, estar dispuesto a equivocarse, pero también a brindar y recibir confianza, por eso todos pueden enseñar, pueden hacerlo en un lugar u otro, recorriendo la ciudad, mostrando lo que saben y afianzando el sentido vital y adaptativo del movimiento.

\section{Los otros: “durar” y perdurar, sujetos en tensión}

En el parkour, algunos de sus practicantes piensan que los demás ciudadanos los visualizan como vándalos y no comprenden la lógica de la práctica - moverse ágilmente en el entorno tal como lo hacen los demás habitantes cuando caminan o se desplazan por la ciudad--, en este sentido, los traceur mencionan que la mayoría de las personas que no conocen sobre sus actividades, relacionan los aspectos estéticos, forma de vestir, práctica de calle y jóvenes como un asunto vinculado al vandalismo, "uno va por ahí y lo ven a uno como descalzurriado, los tenis todos rotos y eso, pues la primera impresión es: este man es un gamín” (EG-PP4-GC, comunicación personal, mayo 5 de 2016), esta situación genera una imagen negativa de la práctica que opaca el sentido que motiva las acciones; sin embargo, los traceurs aclaran que la práctica puede prestarse para forjar habilidades y destrezas con fines negativos como robar o ingresar a lugares prohibidos, por ello creen que es por medio del fortalecimiento de una filosofía de superación, amistad y utilidad social con la que pueden enfrentar este tipo de intenciones y distorsiones.

Tu eres fuerte para un fin, ¿para qué fin? - para ayudar a la sociedad, por eso este entrenamiento era para los bomberos, iquiero ser útil!, ¿para qué? Para durar, no es algo solo para nosotros, la idea es que sirva para los demás. (EI-PP5-GA, comunicación personal, 16 de agosto de 2016)

En otro sentido, algunos practicantes conocidos como fouser hacen parkour para enaltecer el ego propio y no por disciplina, otros asumen el parkour como una práctica competitiva y de rivalidad en la que exaltan las virtudes propias demeritando las ajenas. Por otra parte, algunos miembros de la familia parental de los traceurs - padres, tíos o hermanos mayores-, consideran el parkour como una práctica molesta y que atrae problemas, pues rompe modelos estéticos frente al vestir y expone otras formas de actuar públicamente, “mi mamá no estuvo de acuerdo en la manera en que me estaba vistiendo" (EG-PP3-GA, comunicación personal, 9 de febrero de 2016). Los más jóvenes reclaman a su núcleo familiar confianza y respaldo ante una práctica que puede ser igual de valiosa a cualquier deporte o actividad artística, "siempre fui muy rebelde y por eso fue que empecé a practicar este deporte” (EG-PP2-GC, comunicación personal, 14 de junio de 2016). Sobresale la relación con las madres, su preocupación es evidente frente a la integridad física de sus hijos, dicha preocupación suele compartirse en menor grado con otros miembros de la familia, aquí la edad no importa, todos los entrevistados manifiestan la prevención que sus progenitoras tienen sobre el riesgo que se corre ante las caídas, lesiones, disputas territoriales con otros grupos, tensiones y conflictos con transeúntes y habitantes de 
Parkour y skateboard, intersubjetividad en las calles de la ciudad de Bogotá

los lugares en los que practican, discusiones y peleas con la policía y organismos del estado. Entre los más jóvenes, practicar y exponerse a los riesgos es una forma de establecer cierto grado de autonomía y capacidad de decisión que cuestiona los imperativos del núcleo familiar, situación que al pasar el tiempo conduce a la admiración no solo por la decisión, sino por el cuidado de sí, del otro y de las habilidades y destrezas motrices que desarrollan, "llegó el fin de semana con su mamá, nos contó que tenía limitaciones pero que quería aprender porque veía que nos cuidábamos entre todos" (EG-PP1-GB, comunicación personal, 11 de abril de 2016); luego de llegar a esto, sus familias reconocen los aportes del parkour a la personalidad del practicante, pues este se muestra más seguro, decidido y cuidadoso.

Las relaciones con algunas autoridades y personas residentes de conjuntos cerrados son tensionantes y conflictivas por las formas de tratarles, por ejemplo: reclamos aireados, lenguaje agresivo y grosero, imposición y autoridad, señalamientos, amenazas, entre otros hacen parte de repertorio de estrategias de confrontación que otros ciudadanos usan para hacer valer su presencia y control sobre el espacio, "en ese momento llegaron los policías y nos dijeron que nos daban una hora para irnos del lugar” (EI-PP3-GB, comunicación personal, 7 noviembre de 2016); “toda la gente de esa comunidad nos quitó las llantas que habíamos llevado desde Timiza, esa gente siempre nos ha llevado en la mala” (EG-PP4-GA, comunicación personal, 9 de marzo de 2016). Contrario a esto, también se relacionan con personas y residentes de conjuntos que les facilitan los espacios, les admiran y preguntan sobre la práctica. En un buen número de casos, los vigilantes de conjuntos cerrados y edificios corporativos les permiten usar el espacio de manera velada de tal modo que, si les llaman la atención por no persuadir a los traceurs, estos últimos deben asumir la responsabilidad y librar de culpas al trabajador.

Lo anterior se debe al tipo de relación que se gesta entre los sujetos, la cual depende del uso del lenguaje en el que se destacan los modales y buen trato, situación que se desajusta cuando se relacionan con la policía, pues estos suelen usar términos ofensivos, voz fuerte y expresiones amenazantes que exaltan los ánimos de los practicantes. Parte de esto ocurre porque las autoridades policivas no comprenden el sentido de la práctica, razón por la cual la asocian a otro tipo de actividades partiendo de referentes que han construido sobre otros entornos y expresiones sociales. En este sentido, el pacto y conocimiento de los sujetos, el manejo de un lenguaje claro y alejado de la incitación permite comprender de mejor manera los comportamientos de los practicantes y demás ciudadanos frente al uso del espacio.

\section{Skate: rivales, amigos, parque y calle}

Similar a como ocurre en el parkour, el skateboarding se distingue por el sentido de hermandad y reconocimiento colectivo que se manifiesta al momento de realizar los trucos o de compartir tiempo en la práctica, se alienta y anima a sus compañeros para intentar cuantas veces sea necesario un gesto técnico, de allí que la rivalidad queda relegada a un segundo plano, "yo 
siempre pensé que estos manes no hacían nada, que eran vagos, luego fui acercándome más y pude ver que no era así, así como hay gente envidiosa y mala, hay gente que ha sido como mi familia” (EI.PS3-GC, comunicación personal, 23 de octubre de 2016). Entre practicantes se valora el estilo, pero se invita a practicar otros estilos, pues con estos sueltan y mejoran los aspectos técnicos. Distinto al parkour, cuando compiten, el triunfo es importante, sin embargo, destacan con mayor fuerza la retroalimentación para mejorar los trucos pues se busca aprender de los demás. Por otra parte, los estilos en la práctica no tienen nada que ver con las tensiones que se presentan entre los practicantes, por el contrario, este es un factor de reconocimiento mutuo pues en él se encuentran referentes para la generación de nuevos trucos.
Al skate yo lo quiero mucho, también a los skaters porque hacen parte de mi vida, al que quiera ir a los skate Parks, los skaters se ven como amigos, como familia, no vayan a borrar unos a otros, no hay envidias. (EG-PS4-GD, comunicación personal, 7 de junio de 2016)

Por otro lado, no importa la localización geográfica, al llegar a otra ciudad se reconocen como pares y comparten conocimientos y lugares de práctica. Comparten espacios con otras prácticas como biker y roller con los que acuerdan días de práctica o turnos para usar los espacios o enfrentar los obstáculos, de este modo evitan conflictos entre grupos o practicantes, sin embargo, aunque las rivalidades o disputas no son frecuentes, en ocasiones, algunos practicantes reciben insultos por el mal uso que hacen de los lugares: "Yo fui donde esa gente les dije que dejaran sano el parche que no se metieran con los chinos" (EI-PS2-GB, comunicación personal, 4 de febrero de 2017). Otras tensiones se generan por cuenta de la insuficiencia técnica y de tamaño de los parques. Algunas actitudes como chiflar y maltratar con lenguaje despectivo se manifiestan como forma de rechazo cuando uno u otro practicante hace presencia en lugares ocupados mayoritariamente por miembros de otra práctica. "Esa gente vino a sacarnos que por ellos habían llegado primero y eran más” (EI-PS5-GA, comunicación personal, 17 de noviembre de 2016). Esto también se debe a que los lugares de práctica no fueron diseñados para varias disciplinas, sino que por el contrario, fueron construidos bajo un mismo modelo técnico que entre sus defectos, se materializa en lugares reducidos.

Los espacios con los que cuenta Bogotá son estructuralmente malos, reducidos y pocos, por tanto, para los skaters es mejor patinar en la calle, además, el número de practicantes que estos parques atrae es alto y pasará mucho tiempo antes de contar con la oportunidad para lanzar un truco. Por estas razones, la confluencia de disciplinas y practicantes resulta tensionante, por ello, uno de los acuerdos sociales a los que han llegado con otras prácticas, es definir horarios de uso para cada disciplina durante el día o la semana. 
Parkour y skateboard, intersubjetividad en las calles de la ciudad de Bogotá

Los conflictos con otros ciudadanos se presentan por falta de espacios, por patinar en la calle, por el ruido que generan los golpes de las tablas y por el aspecto estético y de rebeldía frente a otras formas de ser y actuar aceptadas socialmente, "La gente del barrio todos los días tenemos problemas con ellos por el ruido al montar" (EG-PS2-GA, comunicación personal, 16 de febrero de 2016); sin embargo, es importante indicar que en el skate no existe un acuerdo sobre la forma de vestir, pero sí sobre los estilos técnicos circunscritos a espacios específicos, por ello, los estilos asociados a la calle detonan tensiones con otros habitantes de la ciudad, pues dependen del tiempo con el que se cuenta para hacer los trucos en oposición a la aprobación social del lugar; es decir, aprovechando el descuido de las autoridades, la soledad de los espacios, la baja concurrencia de personas como ocurre en los casos de los ingresos a los centros comerciales, calles importantes o, en últimas, negociando el uso del espacio.

\begin{abstract}
Ese día yo fui al barrio, y hablé con la gente de la comunidad para que nos dejaran practicar, les di mi punto de vista, les dije que el hecho de vestirnos así o practicar skate quería decir que éramos vicioso o algo así, al final mucha gente, y pues ha sido más fácil desde que se hizo entender eso. (EG-PS3-GD, comunicación personal, 13 de julio de 2016)
\end{abstract}

Otras tensiones se generan por los daños a la infraestructura pública, especialmente barandas, escaleras y bordes de aceras que deterioran los vecindarios, a raíz de estas afectaciones son señalados como vagos, viciosos o delincuentes, como si se tratara de una invasión territorial en la que se agreden simbólica o físicamente, "llegamos a un parque, eso fue en san Cristóbal sur. Había un grupo de punketos y pues entonces nos tratábamos mal, a veces nos agarrábamos con otros parches, con la policía o gente de los barrios" (EI-PS3-GD, comunicación personal, 18 de enero de 2017). Por ello, los vecinos de diversos sectores recurren a la autoridad policiva utilizando la amenaza para persuadir las acciones de los practicantes, cuando esto no es efectivo, se procede mediante mecanismos represivos como los golpes y traslado a unidades permanentes de justicia. En ellas, las autoridades políticas evaden la problemática y recurren a la autoridad policial desde una perspectiva represiva, en este sentido, es importante mencionar que parte de la mala imagen de la práctica corresponde a hábitos individuales de algunos practicantes que consumen sustancias sicoactivas, roban o realizan actividades que no hacen parte del desarrollo técnico y organizativo del skate; sin embargo, es de destacar que el consumo de estas sustancias durante las prácticas no está asociado a la delincuencia, estas se utilizan como estímulos para fijar las técnicas de movimiento.

Hay personas que les gusta fumar marihuana para montar, que les gusta echarse un juetazo, que les gusta comerse un ácido o cosas así porque de pronto el estado para hacer la actividad les va a dar una sensación diferente. (EG-PS1-GA, comunicación personal, 15 de marzo de 2016) 
En otras situaciones, vecinos y practicantes desarrollan acuerdos comunitarios para la gestión de espacios y recursos para la práctica. Si bien los skaters deterioran algunos espacios públicos de uso común, por otra parte, subsanan el daño, no de forma económica sino valorativa, usando la tabla como medio de transporte amigable con el ambiente.

Los padres y madres de los skaters más jóvenes consideran la práctica como un asunto de hombres en la que la presencia de las mujeres es objeto de señalamiento moral, particularmente porque asumen que en la práctica las mujeres están expuestas a lesiones, golpes, tratos y actitudes agresivas, machistas y morbosas de parte de los practicantes masculinos; sin embargo el coraje femenino frente a estas situaciones, desdibuja el modelo canónico de la delicadeza femenina prevaleciente en otras instancias sociales y a la vez enfrenta activamente la realidad misma de estos hechos; en este sentido, la participación de la mujeres en la práctica toma fuerza, pues entre sus objetivos está lograr un nivel técnico igual o superior a los hombres. Las mujeres suelen ser bien tratadas y acogidas entre grupos mayoritariamente constituidos por hombres. La presencia de mujeres en la práctica es importante porque esto atrae otras practicantes, y en consecuencia, la difusión y crecimiento de la misma. Otro hecho destacable es la inculcación de la práctica a los hijos de los practicantes sin hacer reparos en la condición sexual ni de género de sus hijos.

Frente al conocimiento, inicialmente este es empírico, luego se formaliza al punto de consolidar procesos de capacitación. Cuando el conocimiento sobre la práctica se formaliza, la figura del profesor adquiere mayor sentido para atraer más practicantes y estructurar procesos de enseñanza. En el skate como en el parkour, no sólo se aprenden técnicas, sino otras habilidades como: saber filmar, organizar espacios para la práctica, construir estructuras, tubos, rampas, obstáculos, editar videos y difundir información con la cual interactúan con practicantes de otras ciudades y países; de igual manera la formalización de la práctica en programas o escuelas de formación fomenta premeditadamente y con mayor asertividad valores como la solidaridad, el buen trato, el respeto y el cuidado del otro que les lleva a salir de la zona de confort, no sólo por los vínculos que se establecen con otros practicantes, sino por las transiciones y adaptaciones a las circunstancias que posteriormente enfrentarán cuando salgan a recorrer la ciudad.

\section{Discusión}

Parkour y skate constituyen dos prácticas que guardan una estrecha relación con la ciudad, sus espacios y sus habitantes. La mayoría de los practicantes plantean que sus acciones consisten en realizar movimientos fluidos, atrevidos y creativos, razón por la cual consideran la libertad como principio generador de nuevas formas de actuar, no solo se trata de movimientos sino de relaciones intersubjetivas y con sí mismos. Según Redondo (2011), es vital la preparación del practicante porque no solo se debe pensar en la preparación técnica del cuerpo para enfrentar los obstáculos y retos, sino también en la capacidad mental, es una manera de hacer 
Parkour y skateboard, intersubjetividad en las calles de la ciudad de Bogotá

las cosas, una manera de superar situaciones y problemas de la vida cotidiana, una forma de enfrentar los miedos, de relacionarse con los otros, de reconocer y aprender desde diferencia, "se comprende tanto el pensamiento como acción de sí, conocimiento y cuidado de sí, pero al mismo tiempo cuidado del otro [...] se debe cuidar de sí mismo cuidando de los otros" (Garcés y Giraldo, 2013, p. 191), así, la interacción y relación con los otros y consigo mismo plantea la integración del sujeto a la sociedad, según Daros (2009): "El individuo se incorpora el ritmo y las pautas sociales (socialización). La sociedad, por su parte, lo incorpora y con ello el individuo se vitaliza (expande su vida)" (p. 23).

En este sentido, parkour y skate recogen, reproducen y reinventan el carácter vivencial, sensitivo y motriz de la cotidianidad individual y colectiva no solo en la propia práctica, sino en los espacios comunes de la ciudad, pero también, en toda la compleja red de vínculos que tejen desde su actuar, en términos de Jung (2009) se genera un encuentro con la propia sombra, es decir con aquella proyección del sí mismo que se alimenta del sí mismo de los otros. Según Garcés y Giraldo (2013) “para relacionarse con los otros es necesario tener en cuenta el desarrollo del cuidado de sí. No se puede tener cierto efecto en el otro, si antes no se ha hecho ese trabajo consigo mismo" (p. 191); sin embargo, esta relación va mucho más allá, al ser construida en los espacios de la ciudad resignifica sus locaciones y objetos, en muchas ocasiones convierte los espacios marginalizados o de menor sentido de propiedad, en lugares de práctica con sentido territorial. La ausencia y en ocasiones el exceso de peatones, vecinos y demás agentes dinamiza y reproduce la práctica, es decir, un espacio poco transitado, a veces deteriorado o quizá sin ningún sentido de propiedad por parte de otros ciudadanos, una oportunidad para practicar sin restricciones.

Contrario a lo anterior, un lugar altamente utilizado, en mejores condiciones y bajo el control de otros agentes constituye una oportunidad para disputar el reconocimiento e importancia del sujeto y sus prácticas en el espacio. Sin la resistencia de otros actores, el espacio es transformado por aquellos que lo reclaman en propiedad y bajo los criterios de sus actuaciones; en este sentido, estas prácticas intersubjetivas cumplen un rol social importante, moviliza a sus miembros hacia zonas que por efecto de su presencia empiezan a cobrar valor, es por esta razón que el cuerpo juega un papel transcendental en dichas relaciones toda vez que es la condición por la cual se existe, aparece allí en escena la subjetividad creativa trascendente de Jung (2009), no como materia, sino como intención. No obstante, Foucault (1992) señalará que "los dispositivos y tecnologías de la sociedad disciplinaria definen también al cuerpo como un producto social, insertado en relaciones de poder y dominación a través de mecanismos estratégicos, prácticas discursivas, disciplinas y espacios para docilizarlo” (p. 131). En este sentido y a pesar de los disciplinamientos y dispositivos instaurados para regular el cuerpo, este no deja de constituir un espacio de imaginación activa que confronta las sombras del poder, es decir, que se confronta a sí mismo como trascendencia, hecho que se refleja en las particularidades técnico motrices de los traceurs y skaters, mientras los primeros se subjetivan 
en relación directa con los objetos de la ciudad, los segundos utilizan una persiana -tabla- por cual se filtran distintas experiencias.

Se comprende entonces que hay un vínculo significativo entre la experiencia que se gesta en estas prácticas y la intersubjetividad, en este sentido traceurs y skaters están impregnados del mundo exterior, de este recogen todo aquello que subjetivamente les dispara sus intereses y motivaciones cuando interactúan con los demás, incluso las reflexiones que hacen de sí mismos y como parte del sentido de las relaciones más inmediatas sin importar el medio, por ejemplo, redes sociales, amistades, familia, web y eventos. Por ello, Davidson (2003) señala que:

Las acciones intencionales no pueden aparecer antes que las creencias y los deseos, puesto que una acción intencional se explica mediante las creencias y los deseos que las causan; las creencias no pueden aparecer una a una, ya que el contenido de cada creencia depende de su lugar en el nexo con otras creencias y así sucesivamente. (p. 181)

Dicha interacción de intenciones no ocurre en abstracto y por ello la configuración de los actos y del sentido responde a modelos sociales identificables, en este sentido, tanto traceurs, skaters y demás ciudadanos relacionan su propia existencia e interpretaciones del mundo a partir de hacerse consciente de los otros, movilizan su sentido social y dan lugar a comprender los vínculos y tensiones que pueden aparecer cuando se encuentran en los mismos espacios.

\section{Conclusiones}

Los procesos de subjetivación e intersubjetividad que promueven estas prácticas, forman sujetos que ofrecen formas y expresiones alternativas de relación, en algunos casos tensionantes, en otros, armónicas, y que conducen al reconocimiento del otro en los espacios cotidianos de la urbe y que en la mayoría de los casos son los mismos escenarios en los que circulan, viven, se mueven, aparecen y ocultan los diversos habitantes de la ciudad. Esto es importante porque con ello la intersubjetividad cotidiana opera como marco de reflexión de la vida en la urbe a partir de la regulación de los usos, acciones y significados de los lugares.

Relacionarse con otros reviste dejar al desnudo los impulsos, formas de actuar y razones por la cuales me reconozco como sujeto de vínculos, en este sentido, la presencia de los compañeros de práctica, así como de sus rivales o contradictores, constituye una pieza del marco generador de la práctica. Sin embargo, dentro de las prácticas se identifica una forma especial de relaciones, -horizontales-, en las que los saberes y experiencias valen por su carácter formativo y amistoso y no por el prestigio y jerarquía que otorgan, en este sentido, los momentos de interrelación están circunscritos a la solidaridad, la responsabilidad, la motivación y cuidado del otro. 
Parkour y skateboard, intersubjetividad en las calles de la ciudad de Bogotá

Por su carácter nómada y quizá adverso a las convenciones, y bajo la relativa consideración en la que se asume el espacio público como propiedad colectiva, pero simultáneamente como territorio de nadie, estas prácticas entran en conflicto con aquellos sujetos que previamente han designado algún valor a dichos espacios, lo cual genera y se manifiesta en acciones que desatan disputas territoriales, en las que la calle puede constituir un bien vecinal, un lugar de práctica o un espacio de confrontación. Los procesos de subjetivación e intersubjetividad que promueven estas prácticas forman sujetos que ofrecen formas y expresiones alternativas de relación generadas a partir del encuentro con el sí mismo que se alimenta del sí mismo de los otros, traceurs y skaters proyectan no solo sus propias subjetividades, sino que reflejan algunas formas de aquellos con los que confrontan.

Las relaciones intersubjetivas de estas prácticas desbordan los espacios físicos de la ciudad y empiezan a gestarse en las redes digitales, aprovechadas por los practicantes para participar y constituir una comunidad de adeptos distantes geográficamente pero a la vanguardia del intercambio de información; dicha manera de actuar, permite no solo generar vínculos sino adecuarse con mayor rapidez a los cambios de los contextos, en este sentido, la web funciona no solamente como medio de encuentro sino como contenedora de experiencias que sirven de referente a otros practicantes.

\section{Referencias}

Ascânio, F. (2010). O skate e suas possibilidades educacionais. Motriz: Revista de Educação Física, 16(3), 700-807.

Bermúdez, E. (2008). Roqueros y roqueras, pavitos y pavitas, skaters, lesbianas y gays. El papel del consumo cultural en la construcción de representaciones de identidades juveniles. Revista Latinoamericana de Ciencias Sociales y niñez, 6(2), 616-666.

Blanco, O. (2016). Voces urbanas de Caracas. Repertorio lexicográfico de los skater. Cuadernos de Lingüística Hispánica, (27), 111-136.

Caamaño, C. (2007). El Colonialismo y los Skates, Bikers y Raggas en Limón. Revista de Ciencias Sociales, 3(4), 27-42.

Camino, X. (2008). Reinterpretando la ciudad: la cultura Skater y las calles de Barcelona. Apunts Educación Física y Deportes, 1(91), 54-65.

Camino, X. (2013). La Irrupción de la cultura Skater en el espacio público de Barcelona. Arxiu de Etnografia de Catalunya, (13), 11-38.

Carvalho, R. e Pereira, A. (2008). Percursos alternativos: o parkour enquanto fenômeno subcultural. Revista Portuguesa de Ciência do Desporto, 8(3), 427-440. 
Coffey, A. y Atkinson, P. (2003). Encontrar sentido a los datos cualitativos. Medellín, Colombia: Universidad de Antioquia.

Cornejo, M., Villalobos, A., Cerda, G. y Cuadra, L. (2006). El Skate urbano juvenil. Una práctica social y corporal en tiempos de la resignificación de la identidad juvenil chilena. Revista Brasileira de Ciências do Esporte, 28(1), 39-53.

Cortázar, R. (2015). Skate: más que tablas, ruedas y asfalto. Construcciones identitarias y resignificaciones al espacio urbano, desde la práctica del Skateboarding en Medellín. Medellín, Colombia: Universidad de Antioquia.

Cortes, J., Perea, I. y Samiento, J. (2019). Parkour y movimiento humano. Sentidos y significados de su práctica en Bogotá. Convergencias, 26(79), 1-21.

Cortés, J. y Certuche, J. (2013). Proyecto de investigación: Caracterización sociodemografica del parkour en la ciudad Bogotá. Linea de Investigación Motricidad y Desarrollo Humano. Bogotá, Colombia: Corporación Universitaria CENDA

Cortés, J. y Certuche, J. (2015). Proyecto de investigación: Practicas corporales alternativas en la ciudad de Bogotá. Linea de Investigación Motricidad y Desarrollo Humano. Bogotá, Colombia: Corporación Universitaria CENDA

Daros, W. (2009). El entorno social y la escuela. Rosario, Argentina: Artemisa.

Da Silva, L. e Diniz, N. (2014). O que o skate pode dizer sobre o ensino de geografia? Giramundo, práticas pedagógicas, 1(2), 91-97.

Davidson, D. (2003). Subjetivo, intersubjetivo, objetivo. Madrid, España: Cátedra.

Ferro, L. (2011). De la calle al mundo: configuraciones del graffiti y del parkour y campos de posibilidades urbanas. Lisboa, Portugal: Instituto Universitario de Lisboa.

Foucault, M. (1992). El orden del discurso. Madrid, España: La piqueta.

Garcés, L. y Giraldo, C. (2013). El cuidado de sí y de los otros en Foucault, principio orientador para la construcción de una bioética del cuidado. Discusiones Filosóficas, 14(22), 187-201.

Gutiérrez, R. y Rosales, D. (2011). Parkour como medio motriz. $9^{\circ}$ Congreso Argentino y 4 Latinoamericano de Educación Física y Ciencias. Universidad Nacional de La Plata, La Plata, Argentina.

Hernández,Y. y Galindo, R. (2007). El concepto de intersubjetividad en Alfred Schutz. Espacios Públicos, 10(20), 228-240.

Hoyos, G. (1981). La crítica al positivismo científico en la fenomenología de Edmund Husserl. Revista Extensión Cultural, 9(10), 34-47.

Jung, C. G. (2009). El libro rojo. Buenos Aires, Argentina: Philemon Fundation. 
Parkour y skateboard, intersubjetividad en las calles de la ciudad de Bogotá

Leal, N. (1997). El método fenomenológico: principios, momentos y reducciones. Revista del Departamento de investigaciones Universidad Nacional Abierta de Caracas, 52-60.

Lévinas, M. (1987). De otro modo que ser o más allá de la esencia. Salamanca, España: Sígueme.

López. M. (1994). La intersubjetividad trascendental y el mundo. Enrahonar, (22), 33-61.

Machado, G. (2014). De “carrinho” pela cidade: a prática do skate em São Paulo. Intermeios, $15(2), 185-188$.

Márquez, I. y Díez, R. (2015). La cultura skate en las sociedades contemporáneas: una aproximación etnográfica a la ciudad de Madrid. Revista de Metodología de las Ciencias Sociales, (30), 133-158.

Montoro, S. y Baena, A. (2009). Un nuevo contenido en el área de educación física: el Parkour. Revista Digital de Educación Física, 7(37), 106-124.

Moreira, V. (2004). O Método Fenomenológico de Merleau-Ponty como Ferramenta Crítica na Pesquisa em Psicopatologia. Psicologia: Reflexão e Crítica, 17(3), 447-456.

Patiño. A. (2010). Deportes de acción, alto desempeño y riesgo controlado: skateboarding y downhill. Cali, Colombia: Universidad de San Buenaventura.

Pérez, J. (2013). Desarrollo de las capacidades coordinativas a través del juego: parkour. Revista Digital de Educación Física, (20), 57-66.

Quinteros, C. (2017). El derecho a la ciudad: nuevos ciudadanos, re-significaciones y luchas de los skaters en Viña del Mar. Revista del Departamento de Geografía. FFyH-UNC-Argentina, 5(9), 111-129.

Redondo, C. (2011). ¿Qué es el Parkour?: origen. Habilidades: educación física en primaria como base para esta nueva modalidad deportiva. Revista digital innovación y experiencia, 38.

Rotawisky, J. (2013). Parkour, cuerpos que trazan heterotopías urbanas. Revista Colombiana de Antropología, 49(2), 41-61.

Rotawisky, J. (2015). El arte del extravío: corporalidades y efectos en el parkour. Corpografías, 2(2), 194-211.

Saraví, J. (2007). Jóvenes, Skate y Ciudad: entre el juego y el deporte. Revista Educación Física y Deporte, 26(2), 71-80.

Saraví, J. (2012). Skate, espacios urbanos y jóvenes en la ciudad de La Plata (tesis de posgrado). Universidad Nacional de La Plata, La Plata, Argentina.

Saraví, J. (2014). Cuerpos skaters. Una investigación en la ciudad de La Plata. Memorias II Jornadas Internacionales "Sociedades Contemporáneas, subjetividad y escuela. Universidad de Buenos Aires, Buenos Aires. Argentina. 
Scarnatto, M. y Díaz, J. (2010). De peatón a traceur en una diagonal. El Parkour en la ciudad de La Plata. Nuevas prácticas, patrimonios motrices y formas de socialidad. IV Jornadas de Investigación en Educación Corporal. Universidad Nacional de La Plata, La Plata, Argentina.

Schutz, A. (1932). Fenomenología del mundo social. Buenos Aires, Argentina: Editorial Paidós.

Schutz, A. y Luckmann, T. (1973). Las estructuras del mundo de la vida. Buenos Aires, Argentina: Amorrortu Editores.

Silva, V. (2012). Coletivos juvenis e Parkour no Brasil: percursos interculturais e identidades. Tomo, 5(21), 63-99.

Strauss, A. y Corbin, J. (2002). Bases de la investigación cualitativa. Técnicas y procedimientos para desarrollar la teoría fundamentada. Medellín, Colombia: Universidad de Antioquia.

Vieira, A. e De Sousa, A. (2016). Parkour e valores morais: Ser forte para ser útil. Motrivivência, 28(47), 226-240.

Villagrán, J. y Scarnatto. M. (2013). Itinerarios del cuerpo y la ciudad. El caso del Parkour en la Ciudad de La Plata. $9^{\circ}$ Congreso Argentino y 4 Latinoamericano de Educación Física y Ciencias. Departamento de Educación Física Facultad de Humanidades y Ciencias de la Educación, Universidad Nacional de La Plata, La Plata, Argentina.

Zuluaga, L. M. y Vélez, M. M. (2013). Prácticas de resistencia de jóvenes skaters en la ciudad de Medellín. Medellín, Colombia: Universidad Pontificia Bolivariana. 